\title{
Malignancy-Associated Membranous Nephropathy with Positive Anti-PLA2R Autoantibodies: Coincidence or Connection
}

\author{
Lyle W. Baker ${ }^{a} \quad$ Jaime Jimenez-Lopez ${ }^{b} \quad$ Xochiquetzal J. Geiger ${ }^{c}$ \\ Nabeel Aslam ${ }^{a}$ \\ aDivision of Nephrology and Hypertension, Mayo Clinic, Jacksonville, FL, USA; 'Division \\ of Nephrology and Hypertension, Mayo Clinic Health System, Eau Claire, WI, USA; \\ 'Department of Pathology, Mayo Clinic, Jacksonville, FL, USA
}

\author{
Keywords \\ Membranous glomerulonephritis - Anti-phospholipase-A2 receptor antibodies . \\ Malignancy $\cdot$ Renal cell carcinoma $\cdot$ Nephrotic syndrome
}

\begin{abstract}
Membranous nephropathy ( $\mathrm{MN}$ ) is currently classified as either primary - often associated with positive anti-phospholipase-A2 receptor (PLA2R) autoantibodies - or as secondary associated with malignancy, infection, medications, or autoimmune disease. We present a case of biopsy-proven MN with very high serum titer of anti-PLA2R autoantibodies in a patient with a synchronous diagnosis of poorly differentiated esophageal adenocarcinoma and renal cell carcinoma who presented with nephrotic syndrome. Based on the current classification, $\mathrm{MN}$ in the presence of active malignancy is diagnosed as secondary and unlikely to have positive anti-PLA2R autoantibodies. This raises several questions: whether this patient has secondary MN associated with malignancy and coincidentally discovered anti-PLA2R autoantibodies, primary MN due to anti-PLA2R autoantibodies with coincidentally discovered malignancy, or whether malignancy can induce the formation of anti-PLA2R autoantibodies that result in MN. This case report highlights the importance of age-appropriate cancer screening, even in patients with presumed primary $M N$ and positive anti-PLA2R autoantibodies.
\end{abstract}




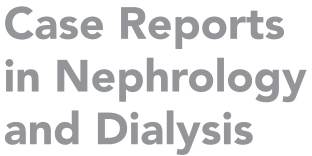

Introduction

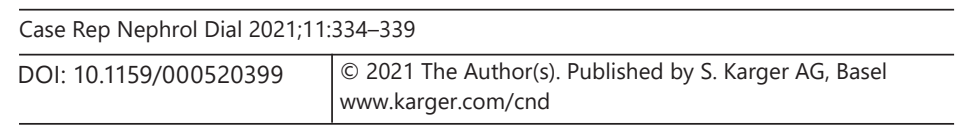

Baker et al.: Malignancy-Associated Membranous Nephropathy with Positive Anti-PLA2R

Membranous nephropathy ( $\mathrm{MN}$ ) is the most common cause of nephrotic syndrome in nondiabetic Caucasian adults, representing as high as $40 \%$ of cases in adults over 60 years of age [1]. MN is characterized by changes in the glomerular basement membrane (GBM) caused by immune complex formation from deposition of immunoglobulin G (IgG) to antigens in between podocytes and the GBM. These immune complexes trigger activation of complement and the membrane attack complex resulting in glomerular injury [1]. Around $75 \%$ of patients with $\mathrm{MN}$ are classified as primary $\mathrm{MN}$, often associated with positive anti-phospholipase-A2 receptor (PLA2R) autoantibodies, with the remaining percentage classified as secondary MN, associated with a secondary cause such as malignancy, infection, medications, or autoimmune disease. Two podocyte antigens have been well established as being involved in the pathogenesis of primary MN: M-type phospholipase-A2 receptor (PLA2R) and thrombospondin type-1 domain-containing 7A (THSD7A) [1]. Autoantibodies to PLA2R have been found in high proportions of patients with primary $\mathrm{MN}$, ranging from 70 to $80 \%$ [2, 3]. We present a case of biopsy-proven MN in a patient with positive serologic anti-PLA2R autoantibodies and 2 simultaneous active malignancies.

\section{Case Report}

A 68-year-old Caucasian male with a history of T3N1 poorly differentiated esophageal adenocarcinoma and a known 5 -cm left-sided renal mass presented with a 3-week history of lower extremity edema. Other past medical history included hypertension treated with ramipril $10 \mathrm{mg}$ daily. The patient had completed 6 cycles of carboplatin and paclitaxel chemotherapy with 7 cycles of radiation for his esophageal adenocarcinoma 3 months before. However, subsequent positron emission tomography scan showed partial response to therapy. He was planning to undergo esophagectomy followed by total left nephrectomy prior to his presentation. He denied nonsteroidal anti-inflammatory drug use. Physical exam showed blood pressure 116/75 mm Hg, pulse 113 per minute, and severe lower extremity pitting edema. Labs demonstrated a serum albumin level of $1.1 \mathrm{~g} / \mathrm{dL}$, serum total cholesterol $251 \mathrm{mg} / \mathrm{dL}$, serum creatinine $1.7 \mathrm{mg} / \mathrm{dL}$ (eGFR of $40 \mathrm{~mL} / \mathrm{min}$ / BSA per CKD-EPI), and a 24-h urine protein $8.3 \mathrm{~g}$. Hepatitis B and C serologies were negative. Serum anti-PLA2R autoantibodies were positive on immunofluorescence (IF) assay and elevated on enzyme-linked immunosorbent assay with an initial value of $695 \mathrm{RU} / \mathrm{mL}$ followed by $>1,500 \mathrm{RU} / \mathrm{mL} 3$ weeks later (negative $<14 \mathrm{RU} / \mathrm{mL}$ and positive $>19 \mathrm{RU} / \mathrm{mL}$ ). Renal biopsy was performed. Light microscopy (shown in Fig. 1) showed 50 glomeruli with 5 globally sclerosed and 21 segmentally sclerosed, slightly increased mesangial matrix with presence of irregular basement membrane spikes and rare small GBM holes. Trichrome stain showed 5-10\% fibrosis. IF showed diffusely global 3+ granular GBM deposition of IgG (shown in Fig. 2), C3, kappa, and 2+ similar pattern for lambda. IgA, IgM, and C1q were negative. Electron microscopy (shown in Fig. 3) showed numerous subepithelial deposits, diffusely effaced foot processes, and areas of basement membrane reaction and early spike formation. Pathology findings were consistent with MN. Paraffin IF for anti-PLA2R was indeterminate on renal biopsy. Malignancy-associated MN was suspected given his history of a left-sided renal mass concerning for renal cell carcinoma and his esophageal adenocarcinoma. Immunosuppression for treatment of MN was held due to active malignancy. Urology felt he was a suboptimal candidate for nephrectomy. Interventional radiology performed biopsy and embolization of the renal mass. Pathology confirmed clear cell renal cell carcinoma. Anti-PLA2R staining on tissue samples of the 
Case Reports

in Nephrology and Dialysis
Case Rep Nephrol Dial 2021;11:334-339

DOI: 10.1159/000520399

c) 2021 The Author(s). Published by S. Karger AG, Basel www.karger.com/cnd

Baker et al.: Malignancy-Associated Membranous Nephropathy with Positive Anti-PLA2R

Fig. 1. Light microscopy: segmental glomerular basement membrane irregular spikes and holes with segmental chunky subepithelial eosinophilic material consistent with immune complex deposits (Jones' silver stain. $\times 600$ ).

Fig. 2. Immunofluorescence: segmental granular to coarse chunky glomerular basement membrane and mesangial staining on frozen section immunofluorescence microscopy (IgG immunofluorescence. ×400).

Fig. 3. Electron microscopy: many small- to medium-sized subepithelial immune complex deposits without substructure. There are areas with basement membrane reaction and early spike formation and no subendothelial deposits. Foot processes are diffusely effaced (electron microscopy, $\times 5 \mathrm{~K})$.
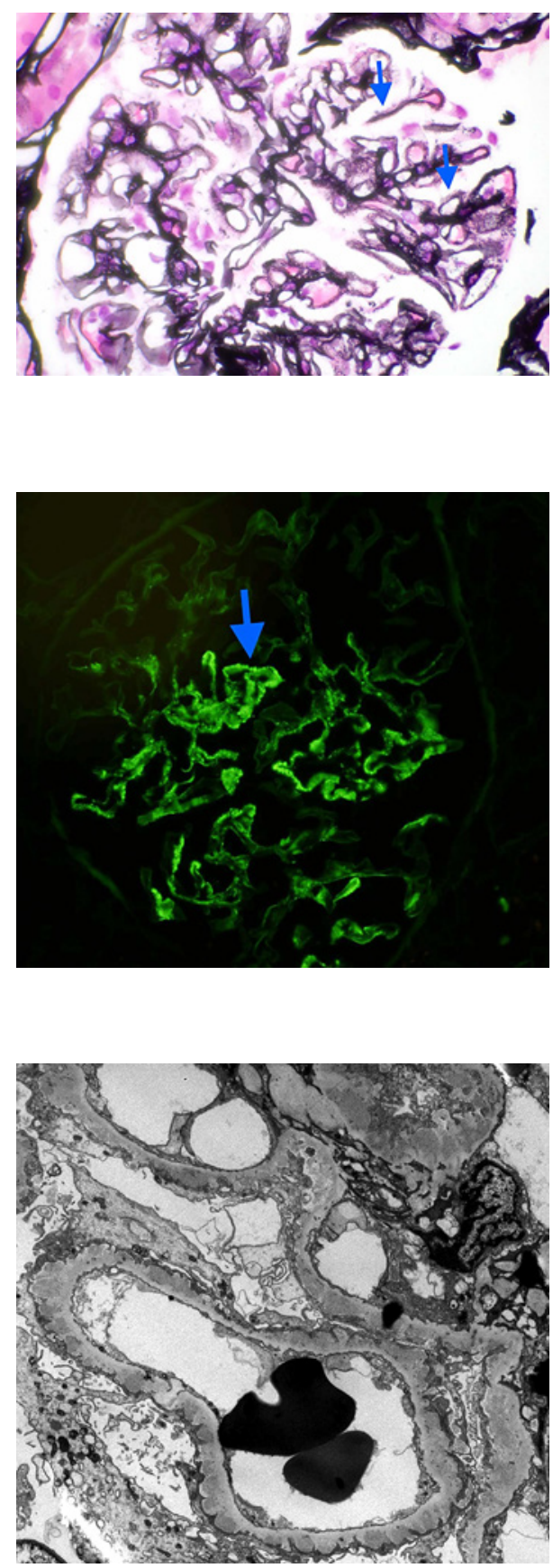

renal cell carcinoma and esophageal adenocarcinoma was both negative. The patient ultimately decided to forgo any additional surgery and was discharged to hospice.

\section{Discussion}

The association between MN and malignancy was described as early as 1966 when it was hypothesized by Lee et al. [4] that tumor may exhibit an antigen that leads to antibody production resulting in antigen-antibody complex deposition in the GBM. Secondary MN has been frequently associated with solid tumors, particularly carcinomas including the kidney, 
colon, lung, and prostate [5]. Prevalence of malignancy is estimated to be 5 times higher in patients with MN than the general population [5]. This has resulted in the accepted recommendation of age-appropriate cancer screening in $\mathrm{MN}$, especially when other secondary causes have been excluded (medications, infections, or autoimmune disease) [6].

Approximately $80 \%$ of MN cases have no apparent secondary causes, resulting in their classification as "idiopathic" or "primary" in nature [5, 7]. The identification of autoantibodies largely associated with primary MN began with the discovery of anti-PLA2R in 2009 and antiTHSD7A in 2014 [8]. Beck's original investigative study found that serum samples from 26 of 37 patients (70\%) with prior "idiopathic" or "primary" classification of MN reacted against a $185-k D$ glycoprotein, identified by mass spectrometry as PLA2R [2]. The proposed explanations for why the remaining population of primary MN were seronegative for anti-PLA2R included the serum samples being collected after patients entered remission with delayed improvement in proteinuria, anti-PLA2R autoantibodies deposited in the kidney before seroconversion, and antibodies other than anti-PLA2R (such as anti-THSD7A) being responsible $[9,10]$. It is relatively uncommon for patients to present with seropositive anti-PLA2R autoantibodies but negative glomerular tissue PLA2R staining, and this finding has been thought to be due to "technical artifacts" [10]. Yet, questions remain whether anti-PLA2R autoantibody production could be provoked by other triggers such as malignancy.

With the reported specificity of anti-PLA2R autoantibodies for MN nearing 100\% [11], and a follow-up analysis performed by Qin et al. [12] demonstrating that anti-PLA2R autoantibodies were largely absent in patients with secondary MN, a common assumption has been made that MN patients with positive anti-PLA2R do not require evaluation for secondary causes of MN. However, Radice et al. [13] found that anti-PLA2R autoantibodies were detectable in 178 of $252(70 \%)$ primary MN patients and 9 of 32 (28\%) secondary MN patients. Recent studies have analyzed cases of secondary membranous nephropathy in patients with hepatitis B, hepatitis $\mathrm{C}$, sarcoidosis, and malignancies and found positive staining for PLA2R in 64\% (25 of 39), 64\% (7 of 11), 75\% (3 of 4), and 70\% (7 of 10) of cases, respectively $[13,14]$. Our patient with biopsy-proven MN had 2 active malignancies and a rising serum titer of anti-PLA2R autoantibodies, an unusual scenario that does not fit the generally accepted classification of MN as being either primary or secondary. This raises the question of whether our patient had secondary MN associated with either of his 2 active malignancies and coincidentally discovered anti-PLA2R autoantibodies, or primary MN due to anti-PLA2R autoantibodies with 2 coincidentally active malignancies. It has been hypothesized that similar cases of secondary MN with positive anti-PLA2R autoantibodies and IgG4-predominant staining may be more physiologically related to primary MN with their associated disease process (neoplasm, autoimmune, and viral hepatitis) being coincidental [14].

Another consideration in this case is whether malignancy can induce the formation of anti-PLA2R autoantibodies that result in MN. To date, there has been no definitive evidence to support a malignancy-mediated production of anti-PLA2R or cross-reactivity with a tumor antigen resulting in anti-PLA2R autoantibodies. Although hypothetically possible, anti-PLA2R staining using paraffin-embedded tissue IF of the renal cell carcinoma and esophageal adenocarcinoma in our patient were both negative. However, sensitivity of IF staining on paraffinembedded tissue in comparison with fresh frozen IF is significantly lower, particularly in primary membranous nephropathy with a sensitivity of only $50 \%$ [15]. Even if we contribute this patient's case to primary MN in the setting of seropositive anti-PLA2R autoantibodies with 2 coincidental active malignancies, it highlights the importance of cancer screening in patients with positive anti-PLA2R autoantibodies consistent with presumed primary MN, since use of immunosuppressive medications can have deleterious effect on tumor growth. More investigation is needed to look for any potential link between anti-PLA2R seropositivity and malignancy. Further studies looking at improvement of proteinuria in patients with

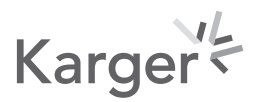


Case Reports

in Nephrology

and Dialysis

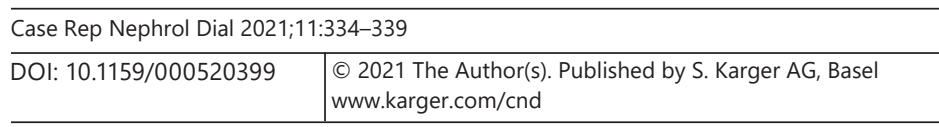

Baker et al.: Malignancy-Associated Membranous Nephropathy with Positive Anti-PLA2R

biopsy-proven MN and positive anti-PLA2R autoantibodies after treatment of an existing secondary cause (such as tumor resection and chemotherapy for malignancy) might provide a better understanding of the role, if any, that PLA2R has on cancer pathophysiology.

In conclusion, we presented a rare case of biopsy-proven $\mathrm{MN}$ with very high serum titer of anti-PLA2R autoantibodies in the setting of 2 active malignancies, an unusual scenario that does not fit the generally accepted classification of $\mathrm{MN}$ as being either primary or secondary. Anti-PLA2R seropositivity should not be considered sufficient to refrain from evaluating for other secondary etiologies. Age-appropriate cancer screening should be pursued in all patients with a diagnosis of MN. An association between malignancy and anti-PLA2R positivity merits further investigation.

\section{Statement of Ethics}

Written informed consent was obtained from the patient's 2 sisters (the patient's only living family) for the publication of this case report and the accompanying images. The patient and the patient's wife are both deceased. The patient has no children. The patient's sisters consented after reviewing the most recent draft of the manuscript and the accompanying images. The completed consent form from the patient's family can be made available to the Editor if needed. Ethical approval was not required for this case report. The Mayo Clinic Institutional Review Board (IRB) acknowledged that based on the responses submitted for this case report through the Mayo Clinic IRBe Human Subjects Research Wizard tool and in accordance with the Code of Federal Regulations, 45 CFR 46.102, the case report does not require IRB review. This letter can be made available to the Editor if needed.

\section{Conflict of Interest Statement}

Drs. L.W. Baker, J. Jimenez-Lopez, X.J. Geiger, and N. Aslam declare that they have no relevant conflicts of interest.

\section{Funding Sources}

No funding was used or needed for the development of this manuscript.

\section{Author Contributions}

All authors (L.W.B., J.J.-L., X.J.G., and N.A.) substantially contributed to the manuscript's conception and design. All authors (L.W.B., J.J.-L., X.J.G., and N.A.) contributed to drafting the manuscript or revisiting it critically for important intellectual content. All authors (L.W.B., J.J.-L., X.J.G., and N.A.) read and approved the final version of the manuscript. All authors (L.W.B., J.J.-L., X.J.G., and N.A.) agreed to be accountable for all aspects of the work.

\section{Data Availability Statement}

All data analyzed during this study are included in this article. Further enquiries can be directed to the corresponding author. 
Case Reports

in Nephrology

and Dialysis

\begin{tabular}{l|l}
\hline Case Rep Nephrol Dial 2021;11:334-339 \\
\hline DOI: 10.1159/000520399 & $\begin{array}{l}\text { (c) 2021 The Author(s). Published by S. Karger AG, Basel } \\
\text { www.karger.com/cnd }\end{array}$ \\
\hline
\end{tabular}

Baker et al.: Malignancy-Associated Membranous Nephropathy with Positive Anti-PLA2R

\section{References}

1 Liu W, Gao C, Dai H, Zheng Y, Dong Z, Gao Y, et al. Immunological pathogenesis of membranous nephropathy: focus on PLA2R1 and its role. Front Immunol. 2019;10:1809.

2 Beck LH Jr, Bonegio RG, Lambeau G, Beck DM, Powell DW, Cummins TD, et al. M-type phospholipase A2 receptor as target antigen in idiopathic membranous nephropathy. N Engl J Med. 2009;361(1):11-21.

3 Glassock RJ. Pathogenesis of membranous nephropathy: a new paradigm in evolution. Contrib Nephrol. 2013; 181:131-42.

4 Lee JC, Yamauchi H, Hopper J Jr. The association of cancer and the nephrotic syndrome. Ann Intern Med. 1966; 64(1):41-51.

5 Bacchetta J, Juillard L, Cochat P, Droz JP. Paraneoplastic glomerular diseases and malignancies. Crit Rev Oncol Hematol. 2009;70(1):39-58.

6 Plaisier E, Ronco P. Screening for cancer in patients with glomerular diseases. Clin J Am Soc Nephrol. 2020; 15(6):886-8.

7 Couser WG. Primary membranous nephropathy. Clin J Am Soc Nephrol. 2017;12(6):983-97.

8 Seitz-Polski B, Lambeau G, Esnault V. Membranous nephropathy: pathophysiology and natural history. Nephrol Ther. 2017;13(Suppl 1):S75-81.

9 van de Logt AE, Hofstra JM, Wetzels JF. Serum anti-PLA2R antibodies can be initially absent in idiopathic membranous nephropathy: seroconversion after prolonged follow-up. Kidney Int. 2015;87(6):1263-4.

10 De Vriese AS, Glassock RJ, Nath KA, Sethi S, Fervenza FC. A proposal for a serology-based approach to membranous nephropathy. J Am Soc Nephrol. 2017;28(2):421-30.

$11 \mathrm{Du} \mathrm{Y,} \mathrm{Li} \mathrm{J,} \mathrm{He} \mathrm{F,} \mathrm{Lv} \mathrm{Y,} \mathrm{Liu} \mathrm{W,} \mathrm{Wu} \mathrm{P,} \mathrm{et} \mathrm{al.} \mathrm{The} \mathrm{diagnosis} \mathrm{accuracy} \mathrm{of} \mathrm{PLA2R-AB} \mathrm{in} \mathrm{the} \mathrm{diagnosis} \mathrm{of} \mathrm{idiopathic}$ membranous nephropathy: a meta-analysis. PLoS One. 2014;9(8):e104936.

12 Qin W, Beck LH Jr, Zeng C, Chen Z, Li S, Zuo K, et al. Anti-phospholipase A2 receptor antibody in membranous nephropathy. J Am Soc Nephrol. 2011;22(6):1137-43.

13 Radice A, Pieruzzi F, Trezzi B, Ghiggeri G, Napodano P, D’Amico M, et al. Diagnostic specificity of autoantibodies to M-type phospholipase A2 receptor (PLA2R) in differentiating idiopathic membranous nephropathy (IMN) from secondary forms and other glomerular diseases. J Nephrol. 2018;31(2):271-8.

14 Larsen CP, Messias NC, Silva FG, Messias E, Walker PD. Determination of primary versus secondary membranous glomerulopathy utilizing phospholipase A2 receptor staining in renal biopsies. Mod Pathol. 2013;26(5):70915.

15 Nasr SH, Fidler ME, Said SM. Paraffin immunofluorescence: a valuable ancillary technique in renal pathology. Kidney Int Rep. 2018;3(6):1260-6. 